\title{
Fast high-resolution micro-CT with exact reconstruction methods.
}

\author{
T Varslot, A Kingston, A Sheppard, A Sakellariou \\ Department of Applied Mathematics, Research School of Physics and Engineering,Australian \\ National University \\ Canberra, Australia
}

\begin{abstract}
We have constructed a helical trajectory X-ray micro-CT system which enables high-resolution tomography within practical acquisition times. In the quest for ever-increasing resolution, lab-based X-ray micro-CT systems are limited by the spot size of the X-ray source. Unfortunately, decreasing the spot size reduces the X-ray flux, and therefore the signal-to-noise ratio (SNR). The reduced source flux can be offset by moving the detector closer to the source, thereby capturing a larger solid angle of the X-ray beam. We employ a helical scanning trajectory, accompanied by an exact reconstruction method to avoid the artifacts resulting from the use of large cone-angles with circular trajectories. In this paper, we present some challenges which arise when adopting this approach in a high-resolution cone-beam micro-CT system.
\end{abstract}

Keywords: helical tomography, exact reconstruction, X-ray tomography, filtered backprojection

\section{INTRODUCTION}

X-ray computed tomography (CT) enables the three-dimensional (3D) imaging of internal structure in an object. A series of projection images are collected of the object at different viewing angles and a reconstruction algorithm is employed to reconstruct the projection data thereby generating a tomogram: a $3 \mathrm{D}$ dataset which is an accurate representation of the structure in the object. X-ray CT is routinely performed on a range of length scales. Micro$\mathrm{CT}$ resides at the lower end of this range, with many applications in material science and biology operating at the resolution limit of current systems. ${ }^{1}$ This limit is currently around $1 \mu \mathrm{m}$. While specialised systems exist that achieve higher resolutions, they are very costly and yet to be proven for quantitative 3D imaging.

There are several difficulties that arise when trying to improve the resolution of lab-based X-ray micro-CT systems. First of all, tomographic reconstruction methods make assumptions about the acquisition geometry. To achieve increased resolution, the tolerance to hardware misalignment decreases. For a reconstruction with cubic voxel size of $1 \mu \mathrm{m}$, small fluctuations in the ambient temperature, or even differential heating of the object due to the X-ray illumination, can produce artifacts and blurring across several voxels in the tomogram. ${ }^{2}$

The second major issue is the close relationship between radiographic resolution, X-ray spot size, and projection data signal to noise ratio (SNR). The spot size is the diameter of the region from which the source produces $\mathrm{X}$-rays. A simple calculation demonstrates how the spot size limits the radiographic resolution in a cone-beam projective geometry. Consider a delta function sample at distance $R$ from a quasi-point source, with a detector and distance $L$, resulting in a geometric magnification factor of $L / R$. Simple ray tracing shows that a source spot of radius $r$ will project the delta function to a blurry spot of diameter $r(L-R) / R$. Demagnifying this back to the sample gives a resolution limit of $r(L-R) / L$. Therefore, for magnification factors $L / R \gg 1$ the spot size is approximately the same as the resolution. Unfortunately, there is a fundamental limit in a conventional bremsstrahlung X-ray tube on the rate at which X-ray photons can be generated per unit area without vaporizing the target material. Therefore, in order to increase the resolution by a factor of two, one must decrease the spot size area, and consequently the maximum X-ray flux, by a factor of four. Now, since the dominant contribution to image noise in a properly configured (i.e. "quantum limited") detector is shot noise arising from finite photon

Further author information: (Send correspondence to T. Varslot)

T. Varslot: E-mail: trond@varslot.net

Developments in X-Ray Tomography VII, edited by Stuart R. Stock, Proc. of SPIE Vol. 7804,

$780413 \cdot$ ? 2010 SPIE · CCC code: $0277-786 X / 10 / \$ 18 \cdot$ doi: $10.1117 / 12.860298$

Proc. of SPIE Vol. 7804 780413-1 
numbers, projection data SNR can be assumed to follow Poisson statistics and be equal to the square root of the number of X-ray photons detected by each pixel. In short, if we wish to preserve SNR while increasing resolution of a given acquisition by factor of two, then we must live with one quarter of the flux, resulting in an acquisition time four times longer. This can lead to unacceptably long experiments, and places strain on stability requirements of the system. It can take 12-24 hours to collect the projection data required to reconstruct a highfidelity tomogram consisting of $2048^{3}$ voxels at $2 \mu \mathrm{m}$ cubic voxel resoluion, ${ }^{3}$ while submicron imaging demands an order of magnitude longer to achieve the same SNR.

In this paper, we look to image at increased cone-angle as a way to reduce acquisition time. Since X-ray tubes generally produce a near-isotropic beam flux over nearly 180 degrees, capturing a larger solid angle of the $\mathrm{X}$-ray beam increases the effective beam flux at the detector. Achieving this higher cone angle is achieved by moving both sample and detector closer to the source, something that's possible with modern transmission style sources that enable one to place samples $400 \mu \mathrm{m}$ from the source spot.

A common setup for X-ray micro-CT facilities is to use a circular orbit scanning trajectory. To generate tomograms, the Felkamp-Davis-Kress (FDK) filtered back-projection algorithm is generally used. ${ }^{4}$ A circular trajectory can be realised using a single rotation stage, and is therefore relatively easy to build with good precision. Furthermore, the FDK algorithm is both robust and tolerant to noise, and can be efficiently implemented on a wide range of computer hardware. However, there are two important limitations with this setup. Firstly, the set of X-ray projections collected using cone-beam illumination and a circular trajectory fail Tuy's sufficiency condition: $^{5}$ they do not contain sufficient information for reconstructing the object. In particular, spatial frequency information that correspond to variation perpendicular to the trajectory plane is missing. This amount of missing information increases for higher cone-angles. Secondly, the FDK algorithm is an approximate reconstruction method outside the trajectory plane. This issue also becomes more pronounced for elevated cone-angles. Combining these deficiencies means that the FDK algorithm can only used when the cone-angle exceeds $\sim \pm 5^{\circ} .{ }^{4}$ Indeed, samples containing features with high vertically-oriented spatial frequencies, such as horizontal planes, cannot even be imaged well at this cone angle. Therefore, for quantitative imaging, it is unwise to approach this limit, meaning that one is obliged to discard the vast majority of the generated X-ray photons.

Several authors have worked on ways to alleviate these issues. In particular, the FDK algorithm has been generalised to provide approximate reconstruction for helical trajectories. ${ }^{6}$ This allows for imaging long objects and potentially overcomes the missing data issue since a helical scanning trajectory satisfies Tuy's sufficiency condition. ${ }^{5}$ Various weighting schemes have also been suggested, to reduce cone-angle artifacts. ${ }^{7,8}$ Despite these extensions, the algorithm remains an approximation, and yield good results only when run at relatively low pitch.

Acquiring projection data along a helical trajectory results in a data set with complete information about the object. ${ }^{5}$ Indeed, exact reconstruction methods for such a case have been known for some time. ${ }^{9-11}$ However, these methods require filtering along the helical axis and therefore do not work well for vertically truncated projection data, uffering from the so-called long object problem. They also require filtering to occur over the boundaries of stitched-together projections, creating very high sensitivity to slight geometric errors or flux variation. The first exact reconstruction method which does not require longitudinal filtering was published for a helical trajectory by Katsevich in 2002. ${ }^{12,13}$ An important ingredient in his work is the Tam-Danielsson window, ${ }^{14}$ which is used to avoid data redundancy, enabling an exact weighting scheme. By virtue of being exact, this algorithm can in principle enable reconstruction from data acquired with arbitrarily large cone angles. Other exact reconstruction methods have since followed, both for helical trajectories ${ }^{15,16}$ and for more general trajectories. ${ }^{17-19}$ Optimisation strategies for exact reconstruction methods have also been reported..$^{20-23}$ Yet, these have been confined to simulation studies, and neglect the practical implementation, particularly issues relating to non-ideal projection data. To our knowledge the exact methods have yet to be successfully deployed in practical imaging applications.

In the current work we present the deployment of the Katsevich method for a micron-resolution X-ray microCT system. The rest of the paper is organised as follows: Section 2 presents the exact reconstruction method of Katsevich ${ }^{13}$ and the Tam-Danielsson window, while Sec. 3 shows how cone-angle and detector size impacts on the total acquisition time. In Sec. 4, we discuss alignment issues which arises when using a helical trajectory. In Sec. 5, we demonstrate imaging capabilities using the exact reconstruction method. Finally, Sec. 6 gives some concluding remarks. 


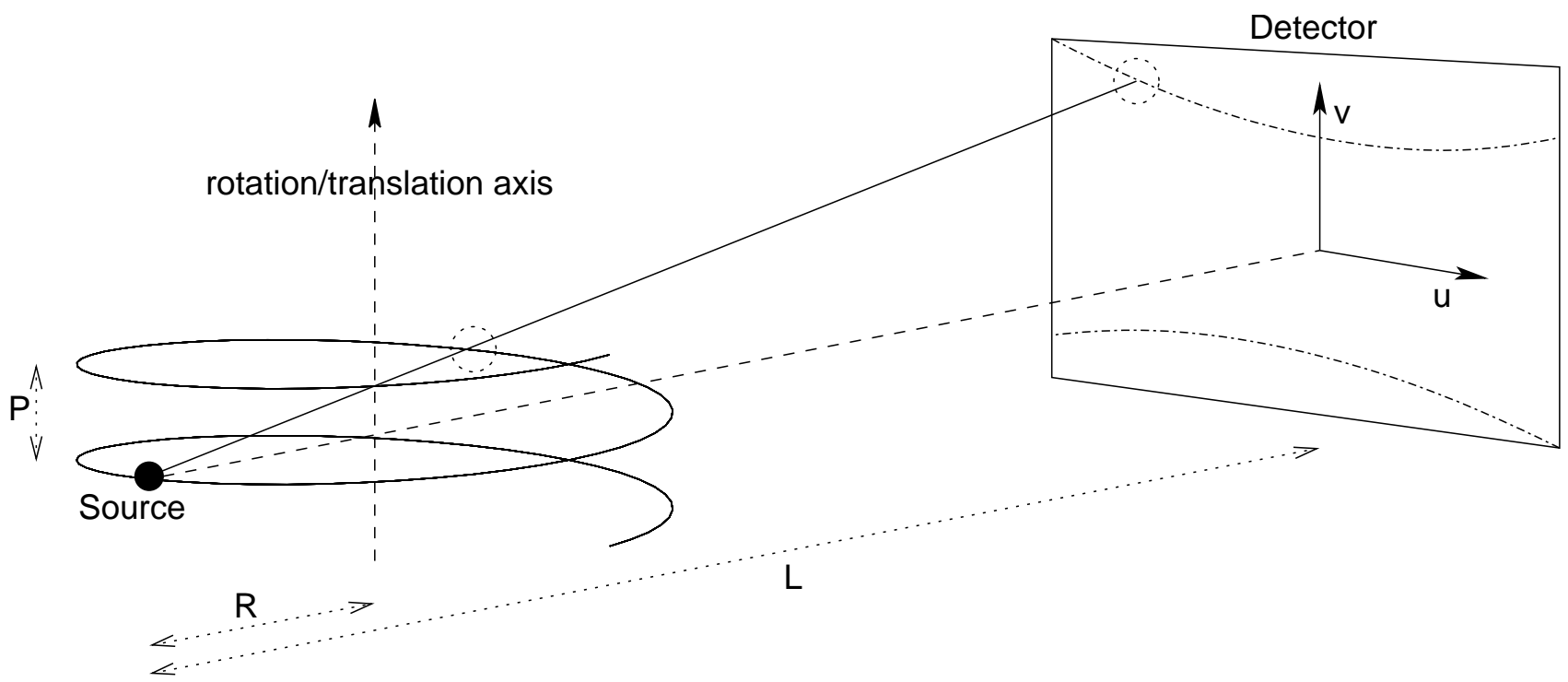

Figure 1. Acquisition geometry for helical cone-beam micro-CT indicating the source, detector and rotation stage positions relative to each other. Important dimensions are sample distance $(R)$, camera length $(L)$ and pitch $(P)$. The solid line from source to detector indicates how the projection of a point on the trajectory prescribes the Tam-Danielsson window. Note that significantly larger cone angle and pitch is potentially attainable with helical scanning, which will further increase its advantage.

\section{RECONSTRUCTION WITH HIGH PITCH}

In this section we will briefly introduce the Katsevich reconstruction method, explain the Tam-Danielsson window, and its implications for the maximal pitch of the trajectory.

Figure 1 depicts a cone-beam X-ray micro-CT acquisition geometry with helical trajectory: the object is placed on a stage with rotation and vertical translation capabilities. For a well-aligned system, we assume that the rotation axis is perpendicular to the detector surface normal, and that the vertical axis of the detector is parallel with the rotation axis. Furthermore, we assume that the detector surface normal which passes through the source also intersects the rotation axis. By necessity, for this to hold, the translation axis of the stage needs to be parallel to its rotation axis. If these assumptions hold, the source trajectory will be a helix of the form

$$
a(\theta)=\left[R \cos \theta, R \sin \theta, \frac{P}{2 \pi} \theta\right]^{\mathrm{T}} .
$$

Here $R$ is the distance from the source to the rotation axis, and $P$ denotes how far upwards the translation stage moves per revolution. The constants $R$ and $P$ are refered to as sample distance and pitch, respectively. Since $P$ denotes how fast we move up along the helix, the total imaging time for a given volume is proportional to the pitch.

The first exact reconstruction method for such a trajectory, which could handle longitudinally-truncated projection data, was proposed by Katsevich ${ }^{12}$ in 2002. Like the FDK method, Katsevich's method is of filtered backprojection type. In fact, implementations can utilize many of the same optimization strategies which have been developed for FDK reconstruction. The difference lies in the filtering of the projection data, and in the carefully chosen subset of the projection data which is backprojected.

In the following we consider a planar detector. Let $(u, v)$ be a coordinate on the detector surface, and let the origin of this coordinate system coincide with closest point on the detector to the source. We denote the distance from the source to the detector by $L$ (see Fig. 1 ). Let the projection dataset be $g(u, v, \theta)$, where $\theta$ is the parameter along the helix which denotes projection angle. Our implementation of the Katsevich method consists of the following main steps, and follows the algorithmic description in ${ }^{24}$ 
1. Compute derivatives of the dataset:

$$
g_{1}(u, v, \theta)=\left(\frac{\partial g}{\partial \theta}+\frac{u^{2}+L^{2}}{L} \frac{\partial g}{\partial u}+\frac{u v}{L} \frac{\partial g}{\partial v}\right)(u, v, \theta) .
$$

2. Weighting of the dataset to account for incident angle of rays onto the detector:

$$
g_{2}(u, v, \theta)=\frac{L}{\sqrt{u^{2}+v^{2}+L^{2}}} g_{1}(u, v, \theta) .
$$

3. Transform the data to a new coordinate system:

$$
\begin{array}{r}
g_{3}(u, \psi, \theta)=g_{2}(u, v(u, \psi), \theta) \\
v(u, \psi)=\frac{L}{2 \pi} \frac{P}{R}\left(\psi+\frac{\psi}{\tan \psi} \frac{u}{L}\right) .
\end{array}
$$

4. Apply a Hilbert-transform along the $u$-direction:

$$
g_{4}(u, \psi, \theta)=\mathcal{H}_{u} g_{3}(u, \psi, \theta) .
$$

5. Transform back to $(u, v, \theta)$-coordinates, i.e., invert the transform in step 3 to obtain $g_{5}(u, v, \theta)$.

6. Backproject the parts of $g_{5}$ which lie inside the Tam-Danielsson window along straight lines intersecting the source location

$$
f(x, y, z)=\int w(x, y, z) g_{5}(p, q, \theta) \chi_{\mathrm{TD}}(p, q) \mathrm{d} \theta,
$$

where $(p, q)$ is the intersection of the line through the source location $a(\theta)$ and $(x, y, z)$ with the detector plane:

$$
p=w[y \cos \theta-x \sin \theta] \quad q=w\left[z-\frac{P}{2 \pi}\right] \quad w=\left(\frac{L}{R}\right)\left(\frac{R}{R+x \cos \theta+y \sin \theta}\right),
$$

and $\chi_{\mathrm{TD}}(p, q)$ is the Tam-Danielsson window.

An important concept here is the Tam-Danielsson window $\chi_{\mathrm{TD}}$. It is the region on the detector which is bounded by the projection of the trajectory segment $a(\theta+s)$ for $s \in[-2 \pi, 2 \pi]$, and masks each projection during the backprojection step to avoid redundant data in our reconstruction. It can be shown that for the helix of Eq. 1, the Tam-Danielsson window is bounded by the curves $\left(u, v_{0}(u)\right)$ and $\left(u, v_{1}(u)\right)$ on the detector

$$
\begin{aligned}
& v_{0}(u)=-\frac{P}{2 \pi R L}\left(u^{2}+L^{2}\right)[\pi / 2+\operatorname{atan}(u / L)] \\
& v_{1}(u)=\frac{P}{2 \pi R L}\left(u^{2}+L^{2}\right)[\pi / 2-\operatorname{atan}(u / L)] .
\end{aligned}
$$

Clearly, a requirement for being able to reconstruct a tomogram from projection data is that the detector height be large enough for the Tam-Danielsson window to fit inside the detector. For a fixed detector height $D_{h}$ and detector width $D_{w}$, this limits the pitch which we may run at

$$
\frac{|P|}{R} \leq \frac{\pi L D_{h}}{\left(\left(D_{w} / 2\right)^{2}+L^{2}\right)\left(\pi / 2-\operatorname{atan}\left(-D_{w} / 2 L\right)\right)} .
$$

Figure 2 shows the Tam-Danielsson window for a detector configuration with $D_{w}=400 \mathrm{~mm}, D_{h}=300 \mathrm{~mm}$ and $L=550 \mathrm{~mm}$. This is typical of the ANU micro-CT system in its development configuration. For a sample distance of $16 \mathrm{~mm}$, coresponding to a field of view $11.6 \mathrm{~mm}$ wide $\times 8.7 \mathrm{~mm}$ high, we can run at a maximal pitch of about $12 \mathrm{~mm}$ per revolution, moving upwards at 1.44 (magnified) detector heights per revolution. We briefly remark that this corresponds to imaging with a cone-angle of approximately $\pm 16^{\circ}$. 


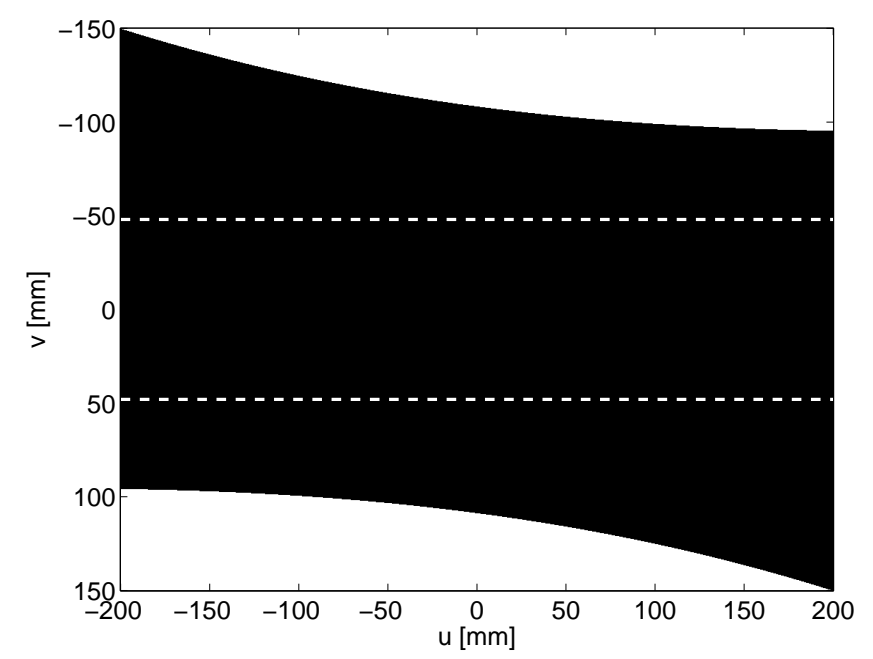

Figure 2. Tam-Danielsson window (black region) corresponding to a pitch $P=12 \mathrm{~mm}$ when using a camera distance $L=550 \mathrm{~mm}$ and sample distance $S=16 \mathrm{~mm}$. We see that the window just fits inside a detector with height $300 \mathrm{~mm}$ and width $400 \mathrm{~mm}$. For comparison, the $\pm 5^{\circ}$ cone-angle restriction is also shown (white dashed rectangle).

\section{TOTAL SCANNING TIME}

The helical trajectory is an open path, and can be used to scan an arbitrary height object. In Fig. 3 we see how the object gradually fades into focus at the top, and then gradually fades out of focus again towards the bottom. The reason for this is that we do not have a complete set of projection data for the end regions. Now, during an acquisition, parts of the object pass in and out of the field of view. To be able to properly reconstruct an object, the projection of each part of the object must enter and exit the Tam-Danielsson window during the scanning run, i.e. the object must be fully out of the Tam-Danielsson window at the start and at the end of the acquisition. The start- and end-projections, where the object is only partially in the field of view, are refered to as overscan. The amount of overscan required for Katsevich's reconstruction method depends on the fan-angle, which is half the angle subtended by the detector at the source in the horizontal plane:

$$
\alpha_{\text {fan }}:=\operatorname{atan}\left(D_{w} / 2 L\right) .
$$

An explicit expression for the overscan required for Katsevich's method is rather complicated and can be found in ${ }^{20}$ it is, however, clear from symmetry that we need the same amount of overscan at either end. This amount starts at $\pi / 2$ for a fan-angle of $\pm 0^{\circ}$, and increases asymptotically to $2 \pi$ as fan-angle approaches $\pm 90^{\circ}$. At $\pm 20^{\circ}$ fan angle, corresponding to the geometry of our test system, the overscan is about a third of a revoluion. In short, we need to make roughly two-thirds of a revolution extra compared to the distance we want to scan. The required number of projections is therefore $N_{\text {scan }}$

$$
N_{\text {scan }} \geq\left(\frac{H}{P}+\operatorname{overscan}\left(\alpha_{\text {fan }}\right)\right) / N_{\text {ppr }},
$$

where $H$ is the desired imaging height, and $N_{\text {ppr }}$ is the number of projections per revolution.

For example, consider scanning a $10 \mathrm{~mm}$ high sample using the geometry described previously of $L=550$, $R=16, D_{w}=400 \mathrm{~mm}$ and $D_{h}=300 \mathrm{~mm}$, with the maximal pitch of $12 \mathrm{~mm} /$ revolution. This requires $\approx 2$ revolutions. In general for this geometry a sample of height $h \mathrm{~mm}$ requires $2 / 3+h / 12$ revolutions. It is apparent that as the sample height becomes greater than its width, the overscan penalty quickly becomes insignificant. For comparison, a circular scan with FDK reconstruction, limited to $\pm 5^{\circ}$ cone-angle, would be able to reconstruct only about $4 \mathrm{~mm}$ of height in a single $360^{\circ}$ scan. More vertical extent can only be achieved by stitching tomograms together, or by using a lower fan angle, with a commensurate loss of flux. 


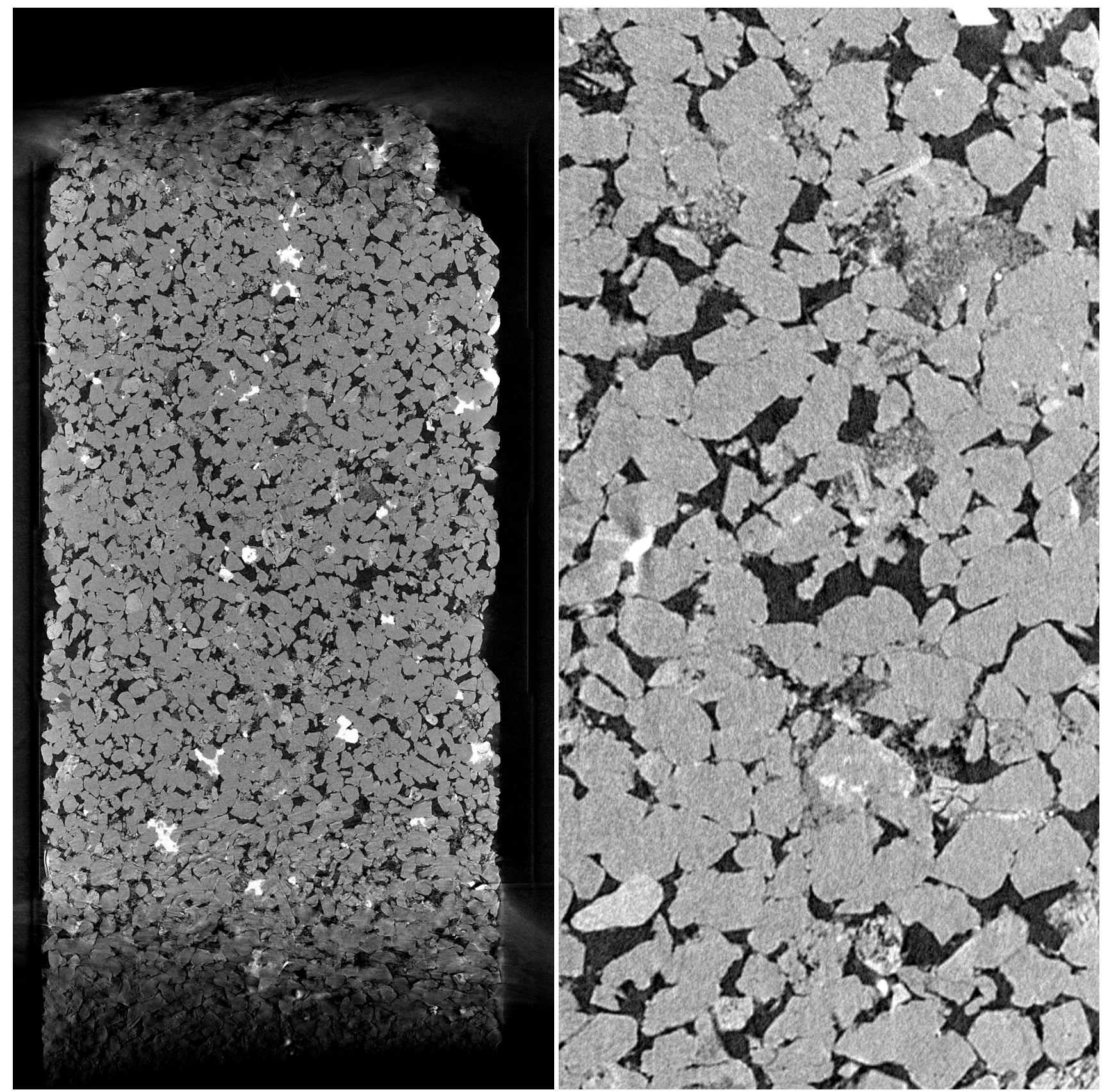

Figure 3. Berea sandstone imaged at $3 \mu \mathrm{m}$ resolution. Incomplete scan towards the top and bottom of the sample cause fading artifacts. 

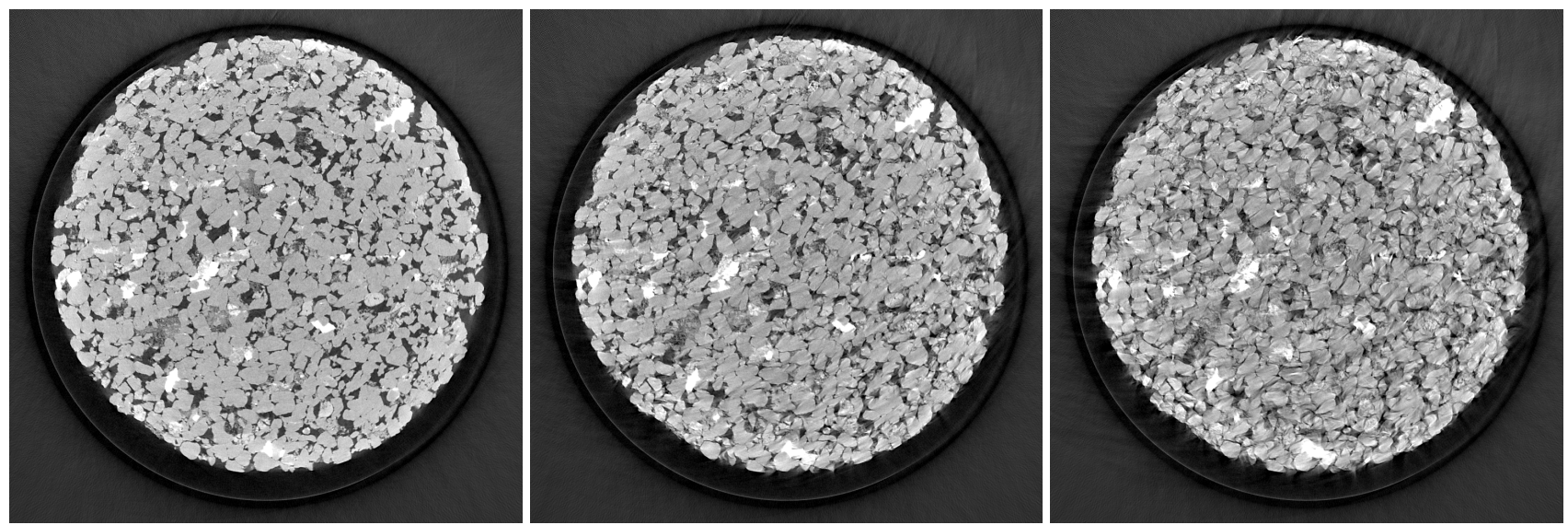

Figure 4. Incorrectly determined sample distance for the Berea sandstone leads to characteristic crests across the sample horizontally. Left: aligned. Middle: misaligned by 1 detector pixel. Right: misaligned by 2 detector pixels.

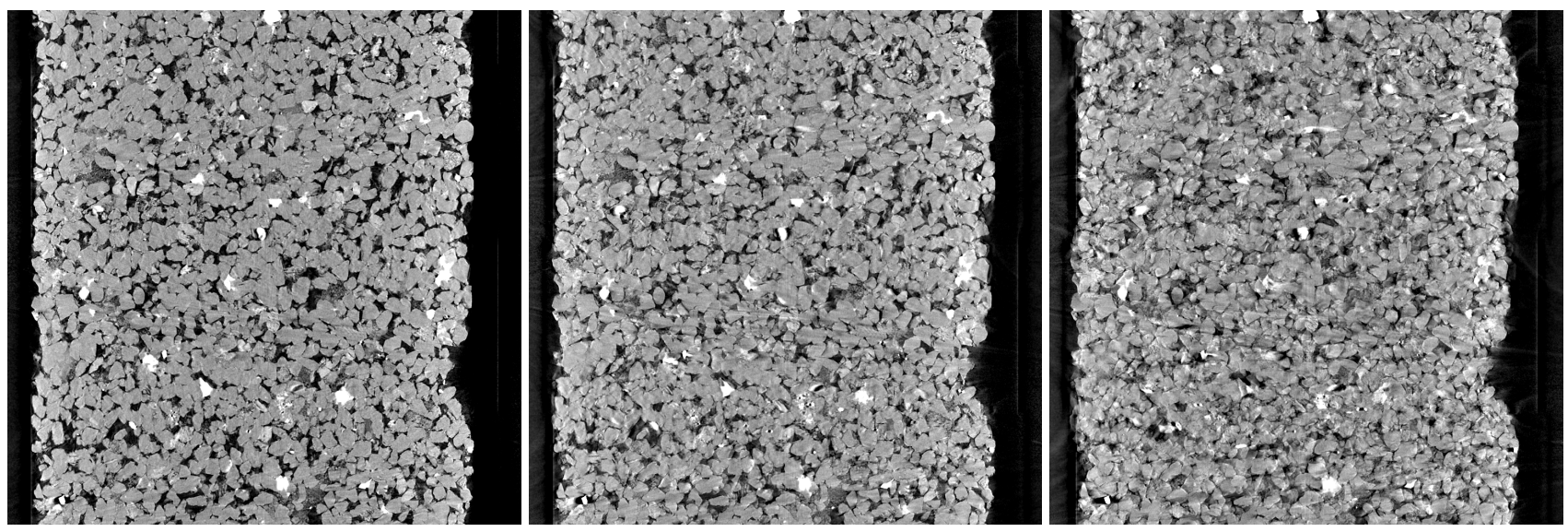

Figure 5. Misalignment of the detector horizontally causes shearing of the sample. Left: aligned. Middle: misaligned by 5 detector pixel. Right: misaligned by 10 detector pixels.

The main point of the preceding discussion is to show that helical scanning, using the maximal pitch that keeps the Tam-Danielsson window in the field of view, is able to efficiently capture a much larger solid angle of $\mathrm{X}$-rays than is possible with FDK methods.

\section{ALIGNMENT}

It is shown in ${ }^{25}$ that for a circular source trajectory, geometric misalignment can be quantified using 6 parameters: horizontal and vertical detector offset, detector in-plane rotation angle, two out-of-plane detector rotation angles, and camera length error. In this case, an error in sample distance will not impact on the overall image quality, but impacts only on the magnification factor (incorrect voxel size).

When using a helical scanning trajectory, three additional parameters can cause misalignment artifacts: two angles describing the direction of the translation axis relative to the rotation axis; and the ratio of pitch over sample distance, $P / R$. We note that in order to get the correct magnification factor, we again need to know the sample distance independently of the pitch. However, in order to obtain a sharp reconstruction, it is the ratio $P / R$ which is of importance (see Eqns.(5) and (9)).

When performing the reconstruction, we make the basic assumption that the translation axis is parallel to the rotation axis. We can therefore compensate for misalignment of the acquisition hardware by mapping each projection onto a virtual detector before reconstructing using an perfectly aligned geometry. An unwanted side effect of this detector mapping operation is an implicit change in the angle associated with each ray. Fortunately this angular change is a higher order effect and can be neglected if the misalignments are sufficiently small. As 
long as the two axis are parallel, the detector mapping is the same for every projection. If the assumption does not hold, the mapping becomes a function not only of the alignment parameters, but also of the projection angle - it changes with position along the helix.

Current experience indicates similar tolerances for misalignment for the original six alignment parameters; a good rule of thumb appears to be that a projected ray through the object has to hit the projection data within 0.5 detector pixels. This also gives us a tolerance for how parallel the translation stage and rotation stage needs to be: for misalignment not to introduce more than $1 \mu \mathrm{m}$ misalignment over $10 \mathrm{~mm}$ of translation ( half a voxel at $2 \mu \mathrm{m}$ voxel size, we require close to $0.1 \mathrm{mRad}$ precision, or 1 part in 10,000.

The need for accuracy in the ratio $P / R$ turns out to be similarly high - better than one part in 1000 , which can mean knowing $R$ to a few microns. Part of the explanation for this is that any changes in $P$ or in $R$ will be magnified by a factor $L / R$ in the projection data. Furthermore, the exact reconstruction method relies on masking out the correct portion of data to backproject at each point, so that the edge effects pairwise cancel out. Artifacts resulting from edge effects in the determination of the Tam-Danielsson window have been reported even on idealised simulation data, and are alleviated by smoothing the window boundary. As seen from Eq.(9), errors in the sample distance or in the pitch will lead to a displacement of the window relative to the projection. As a result, the edge cancellations may fail to give the desired result.

In order to deal with these problems, we have recently developed an auto-focus approach and demonstrated it for use with circular trajectories ${ }^{25}$ which is applicable also for reconstruction from helical X-ray micro-CT projection data. This allows us to obtain the alignment parameters which result in the sharpest possible reconstruction, rather than relying on extreme accuracy in the a priori alignment information. Because of the additional parameters, the search space is larger, so this procedure is more time consuming for helical trajectories.

\section{DESCRIPTION OF IMAGES}

The projection data from which the reconstructions in this paper were made, were acquired on a Varian Paxscan $4030 \mathrm{CB}$ amorphous silicon flat panel detector. This detector has 2048 pixels $\times 1536$ pixels. Each pixel is $194 \mu \mathrm{m}$ $\times 194 \mathrm{\mu m}$ in size; the flat panel is about $400 \mathrm{~mm}$ wide and $300 \mathrm{~mm}$ high. The X-ray source is a GE-Phoenix nanofocus tube, model name xs160t, running a tungsten transmission-type target. This device can produce $\mathrm{X}$-rays from a submicron spot, but for this experiment the spot size was $3 \mu \mathrm{m}$.

In the current development configuration, the detector is fixed at camera length $L=556 \mathrm{~mm}$, while the sample stage can be moved freely between the source and the flat panel in order to adjust the magnification factor.

Fig. 3 shows cross sections of a Berea sandstone. This is a $5 \mathrm{~mm}$ diameter sample, scanned at a sample distance of $8 \mathrm{~mm}$, giving a magnification factor of 69.5 . The resulting cubic voxel size in the tomogram is $2.79 \mu \mathrm{m}$.

Figs. 4 and 5 show artifacts for incorrect sample distance and horizontally misaligned detector, respectively. If the sample distance is off by $0.2 \mathrm{~mm}$, the error is noticable; the crests across the image are an indication that the cancellations are no longer happening the way they should. Note that the projected error resulting from inaccurate sample distance will increase with pitch. This increased sensitivity at high pitch results from the fact that for a ray at angle $\sigma$ to the detector normal, a sample displacement of $\Delta y$ results in a displacement across the detector (i.e. error) of $\Delta y \tan \sigma$. Now, for a ray incident on the edge of the Tam-Danielsson window, $\tan \sigma=P / 2 R$. Therefore, we have less absolute tolerance to errors in the sample distance $R$ when we run at a high pitch $P$. There is a similar increase in sensitivity to errors in detector distance $L$, and to detector tilt, since both these errors are also longitudinal $(y)$ misalignments.

\section{CONCLUDING REMARKS}

We have successfully deployed exact cone-beam reconstruction for helical X-ray micro-CT. This approach has great potential for reducing acquisition time for high-resolution tomograms, as well as the ability to efficiently scan long objects. In fact, we have demonstrated scanning speeds of almost 1.5 detector heights per revolution.

In principle, the reconstruction could be performed without inverting the coordinate transform back to $(u, v, \theta)$-coordinates, and rather backproject the result of the Hilbert transform directly. Though this would 
eliminate one interpolation, the resulting backprojection routine is significantly more cumbersome. By inverting the transform before backprojection we end up with a backprojection which deviates minimally from that used in FDK reconstruction. Hence, all code optimisations for this time-consuming step carry over trivially.

In this work we have only considered the case where the translation axis is parallel to the rotation axis. A situation where this does not hold may be be thought of as a perturbed trajectory, and accounted for by using more general reconstruction methods. In fact, it has been shown that the reconstruction method holds also for perturbed trajectories, as long as they satisfy certain convexity criteria. ${ }^{17}$ Unfortunately, this means that the Tam-Danielsson window will depend on the projection angle. Consequently the optimization strategies, which largely rely on pre-computing Tam-Danielsson window and filtering lines and re-using the result for all projections, are no longer applicable. As long as the perturbations are small, it is still reasonable to re-map the projections onto an ideal geometry, thereby attempting to remove the inconsistency in the projection dataset caused by the trajectory perturbation. The only difference is that for this case the mapping will depend on the location on the source location along the helix.

Future work will explore the possibility to further increase the cone angle.

\section{Acknowledgments}

The authors acknowledge the member companies of the Digital Core Consortium for providing funding support and the Australian National Computing Infrastructure for supplying computing resources. We thank also the Australian Research Council Discovery Program for ongoing research support.

\section{REFERENCES}

[1] Carlson, W., Rowe, T., Ketcham, R., and Colbert, M., "Applications of high-resolution X-ray computed tomography in petrology, meteoritics and palaentology," in [Applications of X-ray computed tomography in the geosciences], Mees, F., Swennen, R., van Geet, M., and Jacobs, P., eds., Special Publications 215, 1-22, Geological Society of London, London (2003).

[2] Sasov, A., liu, X., and Salmon, P., "Compensation of mechanical inaccuracies in micro-CT and nano-CT," in [Proc. of the SPIE], Stock, S. and Stuart, R., eds., 7078, 70781C-70781C-9, SPIE (2008).

[3] Sakellariou, A., Sawkins, T., Senden, T., and Limaye, A., "X-ray tomography for mesoscale phusics applications," Physica A 339, 152-158 (2004).

[4] Feldkamp, L., Davis, L., and Kress, J., "Practical cone-beam algorithm," J Opt Soc Am, 612-619 (1984).

[5] Tuy, H. K., "An inverse formula for cone-beam reconstruction," SIAM J Appl. Math. 43, 546-552 (1983).

[6] Wang, G., Lin, T. H., Cheng, P. C., and Shinozaki, D. M., "A general cone-beam reconstruction algorithm," IEEE Trans. Med. Imaging 12(3), 486-496 (1993).

[7] Yan, M. and Zhang, C., "Tilted plane Feldkamp type reconstruction algorithm for spiral cone beam CT," Med. Phys. 32, 3455-3467 (2005).

[8] Tang, X., Nielsen, R. A., Dutta, S., Samsonov, D., and Hagiwara, A., "A three-dimensional-weighted cone beam filtered backprojection (cb-fbp) algorithm for imag reconstruction in volumetric CT - helical scanning," Med. Phys. 51, 855-874 (2006).

[9] Grangeat, P., Analyse d'un Systèeme d'Imagerie 3D Reconstruction à partir de Radiographies X en Gèomètrie Conique, thèse de doctorat, Ecole Nationale Supèrieure des Tèlècommunications (1987).

[10] Defrise, M. and Clack, R., "A cone-beam reconstruction algorithm using shift-variant filtering and conebeam backprojection," IEEE Trans. Med. Imaging 13(1), 186-195 (1994).

[11] Axelsson, C. and Danielsson, P., "Three-dimensional reconstruction from cone-beam data in $\mathrm{O}\left(n^{3} \log n\right)$ time," Phys. Med. Biol. 339, 152-158 (1994).

[12] Katsevich, A., "Analysis of an exact inversion algorithm for spiral cone- beam CT," Phys. Med. Biol. , 2583-2597 (2002).

[13] Katsevich, A., "Theoretically exact filtered backprojection-type inversion algorithm for spiral CT," SIAM Journal of Applied Math , 2012-2026 (2002). 
[14] Danielsson, P., Edholm, P., and Seger, M., "Towards exact 3D-reconstruction for helical cone-beam scanning of long objects. a new detector arrangement and new completeness condition," in [Proc. 1997 Int Meeting of Fully Three-dimensional Image Reconstruction in Radiology and Nuclear Medicine], Townsend, D. and Kinahan, P., eds., 141-144 (1997).

[15] Zou, Y. and Pan, X. C., "Image reconstruction on PI-lines by use of filtered backprojection," Phys Med Biol 49(12), 2717-2731 (2004).

[16] Zou, Y. and Pan, X. C., "Exact image reconstruction on PI-lines from minimum data in helical cone-beam CT," Phys. Med. Biol. 49(6), 941-959 (2004).

[17] Katsevich, A., Basu, S., and Hsieh, J., "Exact filtered backprojection reconstruction for dynamic pitch helical cone beam computed tomography," Phys. Med. Biol. , 3089-3103 (2004).

[18] Ye, Y. and Wang, G., "Filtered backprojection formula for exact image reconstruction from cone-beam data along a general scanning curve," Med. Phys. 32(1), 42-48 (2005).

[19] Zhao, S., Yu, H., and Wang, G., "A unified framework for exact cone-beam reconstruction formulas," Med. Phys. 32(6), 1712-1721 (2005).

[20] Yan, G., Tian, J., Zhu, S., Quin, C., Dain, Y., Yang, F., Dong, D., and Wu, P., "Fast Katsevich algorithm based on GPU for helical cone-beam computed tomography," IEEE Tran Inf Technology in Biomed (2009). (accepted for publication).

[21] Deng, J., Yu, H., Ni, J., He, T., and Zhao, S., "A parallel implementation of the Katsevich algorithm for 3D CT image reconstruction," J Supercomputing 38(1), 35-47 (2006).

[22] Fontaine, E. and Lee, S., "Optimizing Katsevich image reconstruction algorithm on multicore processors," in [ICPADS '07: Proceedings of the 13th International Conference on Parallel and Distributed Systems], 1-8, IEEE Computer Society, Washington, DC, USA (2007).

[23] Yang, J., Guo, X., kong, Q., Zhou, T., and Jiang, M., "Parallel implementation of Katsevich's FBP algorithm," Int J Biomedical Imaging 2006, 1-8 (2006).

[24] Noo, F., Pack, J., and Heuscher, D., "Exact helical reconstruction using native cone-beam geometries," Phys. Med. Biol. 48, 3787-3818 (2003).

[25] Kingston, A., Sakallariou, A., Sheppard, A., Varslot, T., and Latham, S., "An auto-focus method for generating sharp 3D tomographic images," in [Proc. of the SPIE], 7804, 7804-18, SPIE (2010). (to appear). 